\title{
Predictability and Correlation in Human Metrology
}

\author{
Donald Adjeroh, Deng Cao, Marco Piccirilli, Arun Ross \\ West Virginia University \\ don@csee.wvu.edu, arun.ross@mail.wvu.edu
}

\begin{abstract}
Human metrology provides an important soft biometric, which can be used in challenging situations such as human identification at a distance, when traditional biometric traits such as fingerprints or iris cannot be easily acquired. We study the problem of predictability and correlation in human metrology, using the tools of uncertainty and differential entropy. We show that while various metrological features are highly correlated with each other, there exists some correlation clusters in human metrology, whereby measurements in a cluster tend to be highly correlated with each other but not with the others. Based on these clusters, we propose a two-step approach for predicting unknown body measurements. Using the same framework, we study the problem of estimating other soft biometrics such as weight and gender.
\end{abstract}

\section{INTRODUCTION}

Human recognition and identification using biometrics in unconstrained non-ideal environments is a challenging problem. For identification under confounding situations - such as night-time environments or identification at a distance - most traditional biometric cues such as fingerprints, face or iris may not be readily available. Additionally, in some situations, the poor quality of a video or image may preempt the possibility of reliably deducing identity using face or iris. An alternative is to exploit secondary or soft biometric traits [1] that may be automatically extracted from such poor quality video or images. Metrological features (e.g., body shape, anthropometric measurements, and geometrical features extracted from these measurements) and other soft biometrics (e.g., gait, age, gender, weight, skin texture, etc.) provide a preliminary approach to categorize people in such videos.

In this work, we study the problem of predictability and correlation in human metrology. Our work is closely related to earlier studies on single view metrology [2], [3]; session biometrics using height measurements [4]; and analysis of human body shape and head sizes [5], [6], [7]. Other related work include those on whole-body modeling [8], soft biometrics [1], and analysis of human gait [9], [10]. Our work differs from these in that none of the methods paid any specific attention to whole-body human metrology beyond height or head dimensions. Those based on the CAESAR dataset [6], [5], [7] have all focused on 3D data points. Here, we consider only the $1 \mathrm{D}$ measurements in the CAESAR dataset. The use of $1 \mathrm{D}$ measurements has potential benefits with respect to computation time and automated acquisition from surveillance

WIFS'2010, December 12-15, 2010, Seattle, WA, USA. 978-1-4244-9080-6/10/\$26.00 (c)2010 IEEE. videos. To our knowledge, this is the first attempt at a detailed and formal study of predictability and correlation in wholebody human metrology.

\section{Statistics of Human Metrology}

We first consider the general statistics of human metrology using available data on human body measurements.

\section{A. Datasets}

We used the CAESAR anthropometric dataset that has data pertaining to 2400 US \& Canadian civilians ages 1865 (http://store.sae.org.caesar). There was an equal proportion of male and female subjects, and of people in age ranges 18$29,30-44$ and 45-65. We used 45 human body measurements or attributes (including gender and weight). Measurements are in millimeters, while weight is in kilograms. After data pre-processing and discarding subjects with missing data, we obtained 2369 samples. The first 2000 were used as training samples, and the remaining 369 were used as test samples. We also selected 10 measurements (SET-10M) for closer observation and ease of presentation. The measurements in SET$10 \mathrm{M}$ are as follows 1:arm length; 2:armscye circumference; 3:chest circumference; 4:head breadth; 5:head length; 6:neck base circumference; 7:shoulder breadth; 8:stature; 9: waist circumference; 10:weight. For each body dimension $X$, the individual measurements (the $X_{i}$ 's) are normalized to the $\left[\begin{array}{ll}0 & 1\end{array}\right]$ range using min-max normalization.

\section{B. Statistics}

Table I shows the statistical summary of the measurements in SET-10M using the 2000 people in the training set. Though height (stature) is easier to acquire and has the largest values, it may not necessarily be the best for discriminating between individuals. It has a relatively low coefficient of variation (third to last), and low standard deviation for the normalized values (third to last). The circumferences, which cannot be easily extracted from a 2D video sequence, have higher first order entropy than one dimensional measures such as height and shoulder breadth. This presents a strong case for methods that can predict such body circumferences reliably.

Fig. 1A shows the scatter plots for pairs of measurements in SET-10M. The diagonal plots correspond to the probability densities of individual measurements, while the off diagonals contain the scatter plots for the corresponding pairs. The plots illustrate the nature of the measurements, and the potential dependence and/or correlation between them. Fig. 1B shows the distribution of the ratio of the given measurement to the 
TABLE I

SUMMARY STATISTICS ON HUMAN METROLOGY. *STD2: STD. DEVIATION FOR [0 1] NORMALIZED MEASURES. CV: COEFF. OF VARIATION; CRV COEFF. OF RELATIVE VARIATION (SEE SECTION IV).

\begin{tabular}{|c|c|c|c|c|c|c|c|c|c|c|}
\hline Measure & 1 & 2 & 3 & 4 & 5 & 6 & 7 & 8 & 9 & 10 \\
\hline Min & 237 & 293 & 739 & 123 & 166 & 344 & 346 & 1248 & 557 & 39.9 \\
\hline Max & 416 & 606 & 1574 & 204 & 228 & 598 & 658 & 2084 & 102 & 181 \\
\hline Median & 324 & 415 & 981 & 150 & 194 & 435 & 460 & 1707 & 836 & 73.9 \\
\hline Std & 23.7 & 51.6 & 121.2 & 7.3 & 9.4 & 39.7 & 48.3 & 101.6 & 143.2 & 19.3 \\
\hline${ }^{* S \text { Std2 }}$ & 0.07 & 0.12 & 0.12 & 0.05 & 0.05 & 0.09 & 0.10 & 0.06 & 0.17 & 0.25 \\
\hline CV & 0.13 & 0.17 & 0.15 & 0.09 & 0.11 & 0.16 & 0.16 & 0.12 & 0.13 & 0.14 \\
\hline CRV & 0.13 & 0.17 & 0.15 & 0.09 & 0.15 & 0.16 & 0.16 & 0.12 & 0.13 & 0.14 \\
\hline Entropy & 6.54 & 7.56 & 8.60 & 4.85 & 5.23 & 7.15 & 7.46 & 8.46 & 8.77 & 8.07 \\
\hline
\end{tabular}

head length. (Here, head length is the straight line distance from the glabella to the rearmost point on the skull).

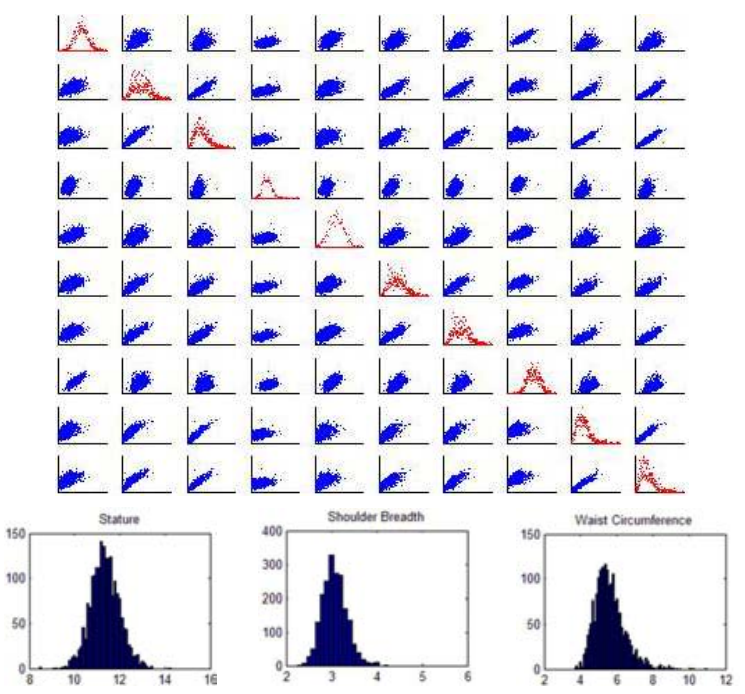

Fig. 1. Statistics of human metrology. Top (A): Scatter plots and probability density for 10 selected measurements; Bottom (B): Ratio plots: Distribution of the number of head lengths contained in other measurements, namely stature, shoulder breadth, and waist circumference.

\section{Correlation in Human Metrology}

\section{A. Correlation}

Our ultimate goal is to build statistical models that can predict human metrology in the event of severe occlusion, missing body parts, poor segmentation, or other problems. From the the principle of Occam's Razor, the smallest model with minimum number of predictors is preferred. Unnecessary predictors will add noise to the estimation process. Too many predictors may introduce redundancy and lead to multicollinearity, with added computational cost. Fig. 1, however, shows that most measurements on the human body are highly correlated. Thus, we study the potential correlation between the measurements, and see how this could be used in building a metrology model.

Given two vectors $Y=y_{1}, y_{2} \ldots, y_{n}$ and $Z=z_{1}, z_{2} \ldots, z_{n}$ the Pearson correlation coefficient is given by $\rho_{Y Z}=$ $\frac{\operatorname{cov}(Y, Z)}{\sigma_{Y} \sigma_{Z}}=\frac{E\{(Y-\bar{Y})(Z-\bar{Z})\}}{\sigma_{Y} \sigma_{Z}}$, where $\sigma_{Y}$ and $\sigma_{Z}$ are the respective standard deviations for $Y$ and $Z$. We computed the correlation coefficient between each pair of measurements in our dataset, forming a $45 \times 45$ correlation matrix. Most measurements have significant correlation with at least one other measurement $\left(\left|\rho_{Y Z}\right| \geq 0.6\right.$ and $p$-value $\left.<0.05\right)$. This points to potential predictability of the measurements based on their correlation partners.

\section{B. Correlation Graph}

To further study the correlation between the measurements, we used the correlation matrix to group the measurements. The correlation matrix was viewed as an adjacency matrix for a graph, where the measurements are considered as nodes in the graph, with the elements in the matrix denoting strength of connection between the nodes. Thus, an edge between any pair of nodes represents some level of correlation between the nodes. Given a threshold on the correlation, we can now construct various graphs such that nodes that are connected in the graph have a correlation coefficient not less than the threshold. We call this the correlation graph. Different thresholds lead to different graphs. In general, with higher thresholds, we get a sparser graph, leading to clusters of nodes that are highly correlated. Lower thresholds lead to more densely connected graphs. Fig. 2 shows an example annotated correlation graph using a theshold of $\left|\rho_{X Y}\right| \geq 0.81$. As can be observed, the measurements generally fall into two basic groups: the 2D group containing mainly circumferences of certain body parts, and the 1D group that contains mainly lengths and heights. Interestingly, the simple correlation measure is able to partition the measurements into various clusters with a physical meaning.

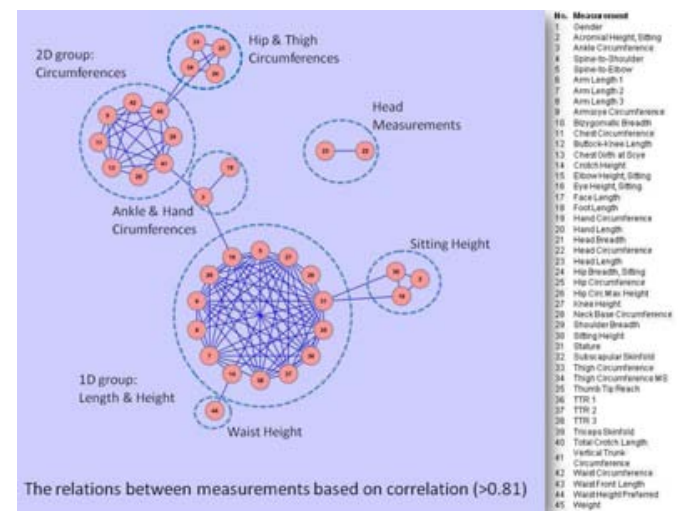

Fig. 2. Correlation clusters in human body measurements based on the correlation graph (at $\left|\rho_{X Y}\right| \geq 0.81$ ). Different thresholds will produce different correlation graphs.

\section{Predictability in Human Metrology}

\section{A. Uncertainty in Metrology}

To investigate the potential predictability of human metrological data, we can use the uncertainty in body dimensions. The issue of uncertainty is related to two important questions with respect to human metrology as a soft biometric: interclass similarity and intra-class variability. These are related to the predictability of such metrological features, their identification capacity or discriminative ability, and their uniqueness. Taken on an individual basis, these measurements may not be very discriminative. But when considered jointly, they can provide a reliable tool for grouping people into several defined 
categories. Here, we focus on the issue of predictability of human metrology. This is more closely related to inter-class similarity rather than intra-class variability.

The variance of a random variable gives an idea of the uncertainty of the variable. We could assess the inter-class variability of the individual measurements using the coefficient of variability $C V(X)$ and the coefficient of relative variability $C R V(X)$, two simple measures related to the variance. For a given random variable $X$, these are defined as follows:

$$
C V(X)=\frac{\sigma_{X}}{\mu_{X}} ; C R V(X)=\frac{\sigma_{X}}{\max \left\{X_{i}\right\}-\min \left\{X_{i}\right\}}
$$

Perhaps, a better approach for studying the uncertainty of a random variable is by use of entropy. Let $X=x_{1}, x_{2} \ldots x_{n}$ be a sequence with symbols from an alphabet $\Sigma$. The entropy of the sequence is defined as:

$$
H(X)=-\sum_{i=1}^{|\Sigma|} p\left(\sigma_{i}\right) \log p\left(\sigma_{i}\right),
$$

where $p\left(\sigma_{i}\right)$ is the probability of the $i$-th symbol in the alphabet, $\Sigma$. Given that our measurements are continuous variables, we can consider the differential entropy rather than the discrete entropy. Let $X$ be be a continuous random variable with probability density function $p(x)$, and support set $\Omega=\{x \mid p(x)>0\}$. Assuming the integral exists, the differential entropy is then given by:

$$
h(X)=-\int_{\Omega} p(x) \log _{e} p(x) d x .
$$

For Gaussian variables this becomes,

$$
h(X)=-\int_{\Omega} p(x) \log _{e}\left(\frac{1}{\sigma \sqrt{2 \pi}} \exp \left(-\frac{(x-\mu)^{2}}{2 \sigma^{2}}\right)\right) d x .
$$

This can be evaluated to obtain $h(X)=\log _{2}(\sqrt{2 \pi e} \sigma)$ bits.

Thus, for Gaussian random variables, the differential entropy becomes a simple function of the variance. For most of the measurements on human body dimensions, the distribution can be approximated as a Gaussian and, hence, the above expression can be used to get an idea of the differential entropy. The joint entropy between two random variables shows the joint uncertainty between the two variables. The joint differential entropy for two continuous random variables $X$ and $Y$, with joint probability density function $p(x, y)$ is given by $h(X, Y)=-\int_{\Omega} p(x, y) \log p(x, y) d x d y$. The conditional differential entropy is given by

$$
h(X \mid Y)=-\int_{\Omega} p(x, y) \log p(x \mid y) d x d y=h(X, Y)-h(Y) .
$$

The mutual information between two random variables tells us how much information one contains about the other. A high mutual information implies some relative redundancy between the variables. The mutual information is given by $I(X ; Y)=$ $\int p(x, y) \log \frac{p(x, y)}{p(x) p(y)} d x d y$. Equivalently, we can write:

$$
I(X ; Y)=h(X)-h(X \mid Y)=h(X)+h(Y)-h(X, Y) .
$$

The differential entropy provides an upper bound on the discrete entropy. The difference depends on the quantization step-size used in the discretization process. In general, using a quantization step-size $q$ to convert a continuous variable to a discrete counterpart, we have the following relation between the discrete entropy and the differential entropy: $H(X)+\log q \rightarrow h(X)$ as $q \rightarrow 0$.

This is particularly important in our problem of human metrology. The probability distributions are likely to be quantized to discrete values before the entropy is computed. It is easy to extend the discussion on entropy of the measurements to higher order models, beyond the second order. Depending on the underlying distribution, some of the integrals involved may not exist. However, in some special cases, it may be possible to obtain closed form solutions for a subset of the quantities. For instance, for multivariate normal variables $X_{1}, X_{2} \ldots, X_{n}$ with mean vector $\mu$, covariance matrix $\Sigma$, and probability density function

$$
p(\mathbf{x})=\left(\frac{1}{(\sqrt{2 \pi})^{n}|\Sigma|^{1 / 2}}\right) \exp \left(-\frac{1}{2}(\mathbf{x}-\mu)^{T} \Sigma^{-1}(\mathbf{x}-\mu)\right),
$$

where $|\Sigma|$ denotes the determinant of matrix $\Sigma$, the differential entropy can be evaluated to get $h\left(X_{1}, X_{2}, \ldots, X_{n}\right)=$ $\frac{1}{2} \log (2 \pi e)^{n}|\Sigma|$ bits. This is important. First, the Gaussian distribution provides an upper bound for differential entropy. Further, with several measurements of the same body dimension from many people, if we can establish that their joint distribution can be approximated by a multivariate normal distribution, we can then compute their differential entropy by considering mainly their covariance matrix. The joint entropy then provides us with some idea on the ability of the measurements in distinguishing people (or at least in grouping people) since, effectively, we will expect that about $2^{h\left(X_{1}, X_{2}, \ldots, X_{n}\right)}$ people can be distinguished using the measurements directly. Table I showed the statistical summary on human metrology including CV, CRV, and discrete entropy, as assessed from the CAESAR dataset. Fig. 3 shows the entropy plots using different number of bins. Waist circumference has the highest entropy with higher number of bins, followed closely by chest circumference. Our analysis showed that while some measurements carry much information about others (high mutual information, e.g., chest circumference and waist circumference), certain pairs of measurements contain very little information about each other (e.g., stature and head breadth).

\section{B. Prediction Models}

We first apply multiple linear regression to the CAESAR data. The general form is as follows:

$$
\left(\begin{array}{c}
y_{1} \\
y_{2} \\
\vdots \\
y_{n}
\end{array}\right)=\left(\begin{array}{cccc}
1 & X_{11} & \cdots & X_{1 p} \\
1 & X_{21} & & X_{2 p} \\
\vdots & \vdots & \ddots & \vdots \\
1 & X_{n 1} & \cdots & X_{n p}
\end{array}\right)\left(\begin{array}{c}
\beta_{0} \\
\beta_{1} \\
\vdots \\
\beta_{p}
\end{array}\right)+\left(\begin{array}{c}
\varepsilon_{1} \\
\varepsilon_{2} \\
\vdots \\
\varepsilon_{n}
\end{array}\right)
$$

where $y$ is the response variable, $X$ is the input (predictor) variable matrix, $\beta$ is the parameter vector, $\varepsilon$ is the error term, $n$ 


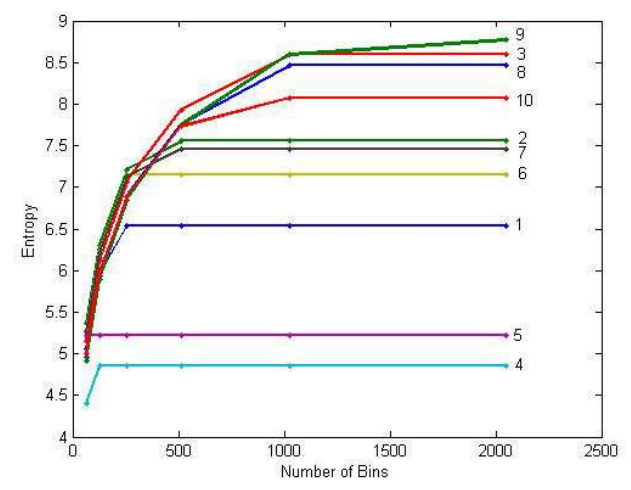

Fig. 3. Entropy plots for the measurements in SET-10M.

is the sample size and $p$ is the number of parameters excluding the constant term. More compactly,

$$
y=\mathbf{X} \beta+\varepsilon
$$

The least-square estimate of $\beta$ will be:

$$
\hat{\beta}=\left(X^{T} X\right)^{-1} X^{T} y .
$$

Since gender is a categorical variable, a logistic regression model is used to predict it separately.

To check the goodness of fit, $R^{2}$, the coefficient of determination is computed as

$$
R^{2}=1-\frac{\sum_{i}\left(\hat{y}_{i}-y_{i}\right)^{2}}{\sum_{i}\left(y_{i}-\bar{y}_{i}\right)^{2}},
$$

which yields a value in the range $0 \leq R^{2} \leq 1$. This gives a measure of the proportion of variability in the response variable that is accounted for by the explanatory variables. We construct a family of prediction models, defined based on the order of the model, the number of variables involved, and the specific way in which the variables are combined. For a maximum order of 2 , with a maximum of 2 predictor variables, this will lead to a family of 31 models, each member denoted by a binary code. For example, we have the following codes for three example members of the family:

2-predictor full model (Model \# 31; code : 111111) :

$$
y=\beta_{0}+\beta_{1} x_{1}+\beta_{2} x_{2}+\beta_{11} x_{1}^{2}+\beta_{22} x_{2}^{2}+\beta_{12} x_{1} x_{2}
$$

1-predictor full model (Model \# 7; code : 110100) :

$$
y=\beta_{0}+\beta_{1} x_{1}+\beta_{11} x_{1}^{2}
$$

2-predictor, partial model (Model \#27, code : 111001) :

$$
y=\beta_{0}+\beta_{1} x_{1}+\beta_{2} x_{2}+\beta_{12} x_{1} x_{2}
$$

Model \#31 produced the overall best results. We validated the models using the normal probability plot and Q-Q plot.

\section{The Human Metrology Predictability Network}

Suppose we have various measurements available, and need to predict one or more other measurements, an important question is how we can choose the best subset of the available measurements to perform the prediction. This is related to the issue of variable selection in pattern recognition [11]. Intuitively, we should choose the subset whose members have maximum correlation with the unknown measurement to be predicted. This can be performed using the correlation graph. To perform prediction for an unknown measurement, say $X$, we involve only the measurements that share some edge with $X$ in the correlation graph, i.e., the members in the subset $X_{C G}=\left\{Y|\tau<| \rho_{X Y} \mid\right\}$, where $\tau$ is the threshold.

The second approach would be to use those measurements that minimize the error when used to predict the unknown measurement. For each measurement $X$, we use the prediction models to perform an initial estimation using the other measurements, and record the resulting error. We then construct a bipartite graph whereby one set of nodes are for the predicted measurements and the other are for the predictors. An edge between a predictor node (say $Y$ ) and a predicted node (say $M X$ ) indicates that the error from the prediction was less than a threshold. Similar to the correlation graph, this also provides a clustering of the measurements, this time based on their predictability.

We repeated the above using the 2-predictor model and generated some interesting networks. Fig. 4 shows an example for a threshold of $M A E \leq 0.04$. The key observation is that, for almost any given measurement, there is a set of pairs of other measurements that can predict it to within the error threshold (usually within 3 hops). In most cases, there are several such pairs. The network thus captures the overall predictability in human metrology. Like the correlation graph, different thresholds will lead to different network configurations.

\section{Cluster-Driven Prediction with PC-Regression}

Expectedly, with more original seed measurements (SMs), we can obtain more accurate prediction. The problem is that for practical situations, we will be able to automatically extract only a few seed measurements. Given the prediction models described earlier, we can use the seed measurements to estimate the remaining measurements, including the difficult-toacquire 2D measures such as the circumferences. Some of the estimated measurements (EMs) will have low MAEs, which means they can be considered as final responses ('patches' for the missing body parts), which can then be used to build a human model. Others may have large errors, and thus their prediction will need to be further refined.

We propose a two-step approach using principal component regression (see Fig. 5). In the first step, we use the regression model to make an initial prediction of the missing measurements based on the seed measurements (SMs). In the second step, we use principal component analysis (PCA) to reduce the number of measurements needed, and then apply the regression model on these features. 


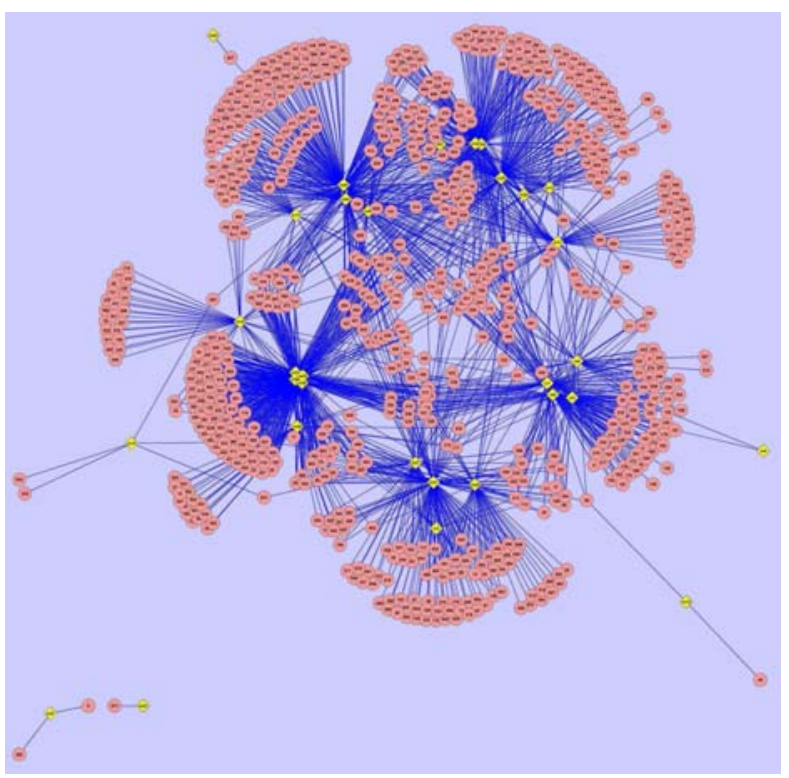

Fig. 4. Predictability network in human metrology. Diamonds correspond to the predicted nodes; circular nodes denote pairs of measurements involved in the prediction. Number in a circular node denotes the index of the measurement pair involved. Results are for the 2-predictor model using a threshold of MAE $\leq 0.04$.

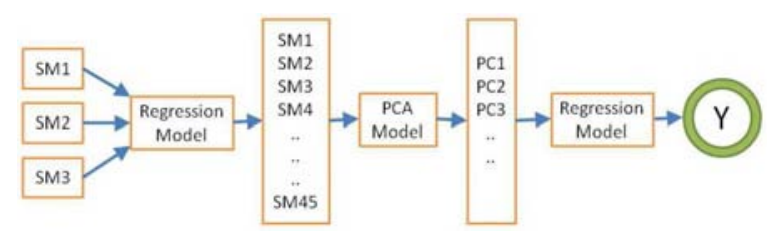

Fig. 5. Two-step prediction, using PC regression, starting with 3 seed measurements.

We use two criteria for variable selection: the principal component regression (PCR) procedure and the traditional adjusted $R^{2}$ criterion. The adjusted coefficient of determination is applied to check the model $R_{a}^{2}=1-\left(1-R^{2}\right) \frac{n-1}{n-p}$. Adding a predictor will only increase $R_{a}^{2}$ if it has some predictive value. In theory we can iteratively apply the PCR untill a certain error threshold is reached. But the problem of overfitting may limit the extent to which we can iterate, and the MAE will typically increase after a few iterations. To improve the prediction precedure, we use a cluster-directed prediction based on the correlation graph. That is, to predict measurement $X$, only a few selected measurements in the subset $X_{C G}=\left\{Y|\tau<| \rho_{X Y} \mid\right\}$ (that is, those that share some edge with $X$ in the graph) are used. We parameterize the prediction using $k$, the number of best partners selected from $X_{C G}$. For the correlation graph, this essentially means the $k$ partners with the highest correlation with $X$. This scheme can be modified to use adjacency information from the metrology predictability network, rather than the correlation graph.

\section{Gender And Weight from Metrology}

The framework described so far can be applied to estimate other soft biometric attributes. This can be used independently for different purposes, or can be combined with the metrological information in a feature space for improved discrimination of individuals. Weight and gender are particularly of interest, given that these are relatively easy for the human to determine and, hence, can easily be used along with other soft biometrics.

Since weight is a continuous variable, we can treat it just like other measurements. From the correlation graph of Fig. 3 , we can observe that weight (measurement \#45) forms an almost connected component with a number of 2D measurements such as thigh circumference and waist circumference. Many other measurements are also correlated with weight. Since gender is a categorical variable, we use the logistic model $f(z)=\frac{e^{z}}{1+e^{z}}, z=\beta_{0}+\beta_{1} x_{1}+\beta_{2} x_{2}+\ldots+\varepsilon$, where $f(z)$ is the probability of obtaining a specific categorical outcome.

\section{RESUlTS}

We ran experiments using the CAESAR dataset described in section 2.1. To test the results on video data, we used the data of 23 individuals from the CMU motion capture (Mocap) database. These already have the dimensions of certain body parts (based on sensors at key landmarks) and we can use them to validate the prediction models developed. Not all measurements in CAESAR were available in the Mocap dataset. We selected 4 measurements, viz., \#8: arm length from shoulder to elbow; \#27: knee height; \#29: shoulder breadth; and \#31:stature (standing height); (see Fig. 2 for CAESAR measurement IDs).

\section{A. Prediction Results}

Fig. 6 shows the variation of prediction performance with $k$, the number of best partners in the cluster-directed prediction. The figure shows that the overall minimum MAE was obtained at $k=3$, while the overall best MAE was obtained at $k=2$. Table II shows the overall performance of the proposed prediction schemes using different measures of prediction performance. The results are based on the cluster-directed prediction model using the correlation graph with best $k=2$ correlated partners.

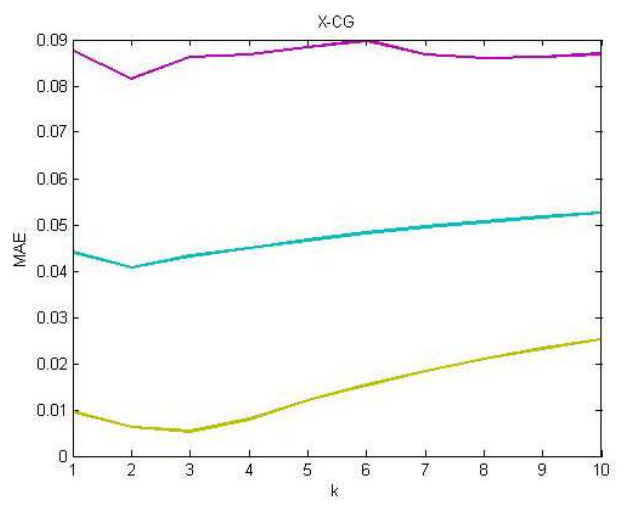

Fig. 6. A: Prediction performance on CAESAR dataset (average for all measurements using $X_{C G}$ ). Top: max MAE; Middle: mean MAE; Bottom: min MAE. Similar results were obtained using clustering based on MAE. 
TABLE II

OVERALL PREDICTION PERFORMANCE OF PROPOSED METHOD

\begin{tabular}{|c|c|c|c|c|}
\hline & $R^{2}$ & $R_{a}^{2}$ & MSE & MAE \\
\hline \hline mean & 0.81198 & 0.811508 & 0.003438 & 0.040792 \\
\hline max & 0.99594 & 0.99593 & 0.010446 & 0.081565 \\
\hline min & 0.40062 & 0.39912 & $6.96 \mathrm{E}-05$ & 0.006198 \\
\hline std & 0.146531 & 0.146898 & 0.002891 & 0.020161 \\
\hline weight & 0.92537 & 0.92518 & 0.001391 & 0.028113 \\
\hline
\end{tabular}

\section{B. Results for Weight and Gender}

The last row in Table II shows the overall performance of the proposed models in weight estimation. Using all the 44 measurements to predict gender resulted in a perfect classification. Using the $3 \mathrm{SMs}$ to predict the other 41 measurements and then using both predicted and original measurements for gender prediction produced a classification rate of $89.7 \%$ on the training set, and $88.9 \%$ for the testing set. With clusterdirected prediction on gender we obtained classification rates of $91.9 \%$ (training), and $90.5 \%$ (testing) for $X_{C G}$ and $93.3 \%$ (training), and $91.0 \%$ (testing) for $X_{M A E}$.

\section{Discriminability of metrological features}

To evaluate the proposed prediction methods, we randomly selected 30 individuals from the CAESAR dataset and 23 people from the CMU Mocap dataset. Then using two seed measurements (SMs), namely, shoulder breadth and stature, we estimated three other measurements. We then computed ratio features (such as those shown in Fig. 1), and formed a 14 dimensional feature space using the SMs, the predicted measurements, and the ratio features. Fig. 7 shows the position of each individual in 3D feature space, using the first three principal components (PCs). The individuals are very well separated showing the ability of the metrological features to discriminate between people. We have used 3 PCs for ease of visualization. Typically more PCs will be used in comparing individuals.

\section{DiscusSiOn AND CONCLUSION}

We have investigated the issue of predictability and correlation in human metrology using information theoretic notions of uncertainty and entropy. It was observed that human body measurements are often correlated. At a given correlation level, the measurements tend to form a kind of cluster, whereby members in a cluster are much more correlated with each other, than with those outside the cluster. When considered from the view point of prediction models, these clusters result in a form of predictability network between human measurements, whereby the measurements are connected to others that can predict them to within a stated error threshold. Using these clusters, we proposed a two-step prediction algorithm for estimating unknown body measurements, with encouraging results. An interesting problem will be to use methods of information network analysis [12], such as between-ness, centrality or network dissociativity measures to characterize the predictability network in human metrology. The discussion on differential entropy can be carried further, for instance, to study the true capacity of metrology in human identification.

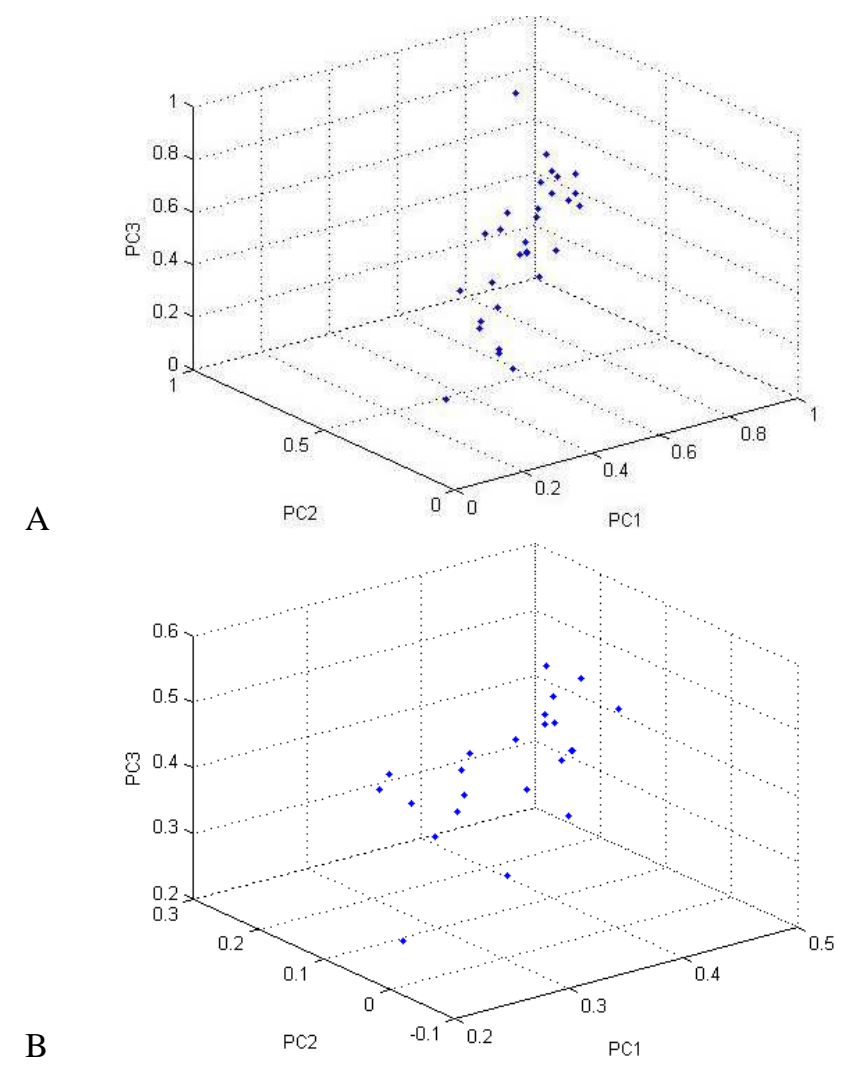

Fig. 7. Discriminating between people using predicted measurements. Given: shoulder breadth \& stature; Estimated: arm length, waist circumference, and head length. (A): Using CAESAR dataset. (B): Using CMU Motion capture dataset. Each point denotes an individual.

\section{REFERENCES}

[1] A. K. Jain, S. C. Dass, and K. Nandakumar, "Soft biometric traits for personal recognition systems," ICBA, pp. 731-738, 2004.

[2] A Criminisi, I. Reid, and A. Zisserman, "Single view metrology," Int'l J. Computer Vision, vol. 40(2), pp. 123-148, 2000.

[3] F. Guo and R. Chellappa, "Video metrology using a single camera," IEEE TPAMI, vol. 32(7), pp. 1329-1335, July, 2010.

[4] C. Madden and M. Piccardi, "Height measurement as a session-based biometric," Proceeding, Image and Vision Computing Workshop, 2005.

[5] B. Allen, B. Curless, and Z. Popović, "The space of all body shapes: reconstruction and parameterization from range scans," $A C M$ Transactions on Graphics (ACM SIGGRAPH 2003), vol. 22(3), no. 8, pp. 587-594, 2003.

[6] A. Godil and S. Ressler, "Retrieval and clustering from a 3D human database based on body and head shape," SAE Digital Human Modeling, 2006.

[7] A. O. Bălan and M. J. Blake, "The naked truth: Estimating body shape under clothing," ECCV, LNCS 5303, pp. 15-29, 2008.

[8] A. Hilton, B. Beresford, T. Gentils, R. Smith, W. Sun, and J. Illingworth, "Whole-body modelling of people from multiview images to populate virtual worlds," The Visual Computer, vol. 16(7), pp. 411-436, 2000.

[9] M. S. Nixon, R. Chelleppa, and T. Tan, Human Identification Based on Gait, Springer, 2006.

[10] M. A. Hossain, Y. Majihara, J.-Q Wang, and Y. Yagi, "Clothing invariant gait identification using part-based clothing categorization and adaptive weight control," Pattern Recognition, vol. 43(6), pp. 2281-2291, 2010.

[11] M. Nadler and E. P. Smith, Pattern Recognition Engineering, Wiley, New York, 1992.

[12] Mark Newman, Albert-Laszlo Barabasi, and Duncan J. Watts, The Structure and Dynamics of Networks, Princeton University Press, 2006. 